\title{
The Electronic Nose: The Effect of Metal Oxide Modifications to Well-Aligned ZnO Nanowire Arrays for Highly Sensitive and Selective Gas Detection
}

\author{
Haiqiao Su, Jiajun Chen, Kai Wang, Alaoddin Ahmad Ayyad and Weilie Zhou \\ Advanced Materials Research Institute (AMRI), University of New Orleans, New Orleans, LA 70148 \\ wzhou@uno.edu
}

\begin{abstract}
:
To mimic biological olfactory receptor arrays, a three dimensional (3D) "electronic nose" was fabricated by using well-aligned $\mathrm{ZnO}$ nanowire arrays modified with different metal oxides. Metal oxide nanowire arrays share 3D structures similar to mammalian olfactory receptor arrays, with thousands of vertically aligned nanowires providing a high reception area which can significantly enhance the sensors' sensitivity. Meanwhile, with different material decorations, each array of nanowires can produce a distinguishable response for each separate analyte, which would provide a promising way to improve the selectivity. We have successfully fabricated gas sensors based on $\mathrm{SnO}_{2}, \operatorname{In}_{2} \mathrm{O}_{3}$, and $\mathrm{WO}_{3}$ coatings which have discriminated five gases which are $\mathrm{NO}_{2}, \mathrm{H}_{2} \mathrm{~S}, \mathrm{H}_{2}, \mathrm{NH}_{3}$, and $\mathrm{CO}$. The sensitivity to $\mathrm{NO}_{2}$ and $\mathrm{H}_{2} \mathrm{~S}$ has reached down to the ppb level.
\end{abstract}

Key words: Electronic nose, ZnO nanowire, Metal oxide, Gas sensor arrays.

\section{Introduction}

Highly sensitive and selective chemical agent detectors have important applications in the areas of environmental emission control, public security, automotive applications, workplace hazard monitoring, medical diagnosis, and so on [1-2]. Among most of the detection technologies, including conductive polymer sensors, metal oxide conductometric sensors, and microcantilever sensors, metal oxide conductometric sensors comprise a significant part of the gas sensor component market. The reason is that metal oxides possess a broad range of electronic, chemical, and physical properties that are often highly sensitive to changes in their chemical environment. As nanotechnology has developed, metal oxide nanostructures have attracted much attention for applications in highly sensitive and selective gas sensors due to their high aspect ratio, large surface area, and availability of versatile structures as compared to their thin film counterparts [3-4]. For example, in gas sensors formed with individual $\ln _{2} \mathrm{O}_{3}$ nanoparticles, a nanowire or nanowire networks can detect $\mathrm{NO}_{2}$ down to the ppb level at room-temperature [5$6]$.

For the purpose of mimicking the biological olfactory system with a diversity of receptor arrays, vertically aligned 3D nanowire arrays have great potential for sensor applications, due to their extremely large surface area which provides a much stronger capability of capturing target molecules in the atmosphere [7-8]. To realize multiple receptor arrays, different metal oxides were coated onto well-aligned $\mathrm{ZnO}$ nanowire arrays, which can be readily grown by hydrothermal synthesis [8-9]. The electrical and gas-sensing properties of individual nanowire arrays were characterized. The electronic nose was fabricated with $\mathrm{ZnO}$ nanowire arrays decorated with the different metal oxides $\left(\mathrm{SnO}_{2}\right.$, $\mathrm{In}_{2} \mathrm{O}_{3}$, and $\mathrm{WO}_{3}$ ) and the gas discrimination of this electronic nose was studied in detail.

\section{Experimental}

The fabrication of vertically aligned semiconductor core-shell nanowire arrays generally starts with well-aligned $\mathrm{ZnO}$ nanowires grown by hydrothermal synthesis [10]. Fig.1 shows the schematic of the fabrication procedures for 3D sensor devices. The substrate was cleaned by a standard process, followed by the well aligned growth of $\mathrm{ZnO}$ nanowire arrays by hydrothermal synthesis [8]. Common thin film techniques, such as sputtering, PLD, or CVD, can be used to deposit the shell layers [8]. In this experiment, $50 \mathrm{~nm} \mathrm{SnO} 2, \ln _{2} \mathrm{O}_{3}$, and $\mathrm{WO}_{3}$ were sputtered in 
a Lesker PVD75 system and a metal mask was employed to ensure selective deposition. Finally, the top electrodes connecting the nanowire arrays were prepared by microfabrication processes [8].
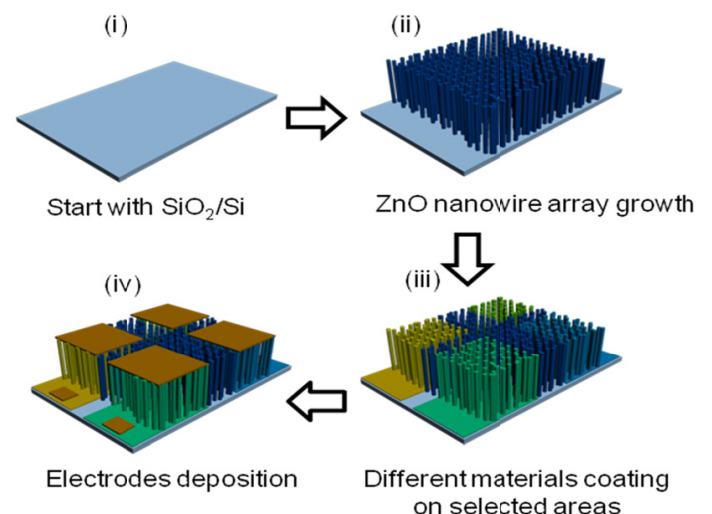
of metal oxide coatings on $\mathrm{ZnO}$ nanowire arrays for selective detection. (i) The procedures start with a cleaned substrate; (ii) Followed by well aligned $\mathrm{ZnO}$ nanowire array growth; (iii) Different material coatings on selected areas; (iv) Electrode deposition.

To test the sensor performance, the nanowire array sensors were loaded onto the gas sensor testing system and the conductance for all the devices was continuously monitored when different air-diluted chemicals were introduced. The devices were connected by $0.02 \mathrm{~mm}$ gold wires and silver paste. The Keithley 2400 source measurement unit cooperating with a 7001 switch module can continuously monitor the conductivity of all the nanowire array devices. The gas sensors were recovered by flushing with a constant dry air flow.

\section{Results and Discussion}

Fig.2 (a) and (b) shows FESEM images of $\mathrm{ZnO}$ nanowire arrays. The pristine $\mathrm{ZnO}$ nanowires have $100 \sim 400 \mathrm{~nm}$ diameters and are $5 \mu \mathrm{m}$ in length.
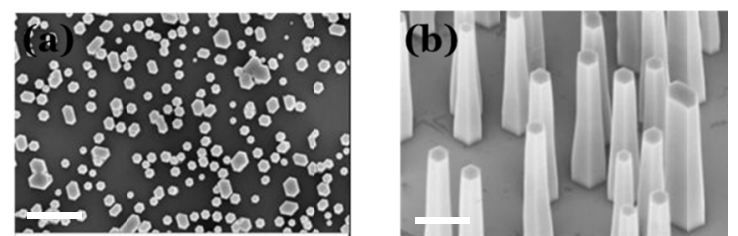

Fig. 2. FESEM images. (a) Top view; (b) View by $30^{\circ}$ tilting. (Scale bar is $1 \mu \mathrm{m}$.)

After different metal oxide coatings, I-V characteristics were obtained when the sensor arrays $\left(\mathrm{ZnO} / \mathrm{SnO}_{2}, \mathrm{ZnO} / \mathrm{In}_{2} \mathrm{O}_{3}\right.$, and $\left.\mathrm{ZnO} / \mathrm{WO}_{3}\right)$ were loaded into testing systems. The linear I-V plots in Fig. 3 indicate that the contact between the electrodes and nanowires are all ohmic.

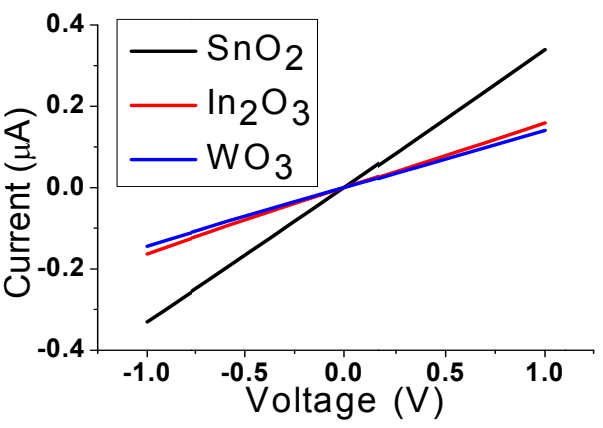

Fig. 3. I-V curves for nanowire arrays coated by different metal oxides.

The sensing responses were measured with air-diluted $\mathrm{NO}_{2}, \mathrm{H}_{2} \mathrm{~S}, \mathrm{NH}_{3}, \mathrm{CO}$, and $\mathrm{H}_{2}$ of different concentrations at room-temperature. All normalized sensing responses, $\left(\mathrm{G}_{\mathrm{gas}}-\mathrm{G}_{\text {air }}\right)$ / $\mathrm{G}_{\text {air }}$, are plotted in Figure 4 (a-e). The devices show high sensitivity to $\mathrm{NO}_{2}$ and $\mathrm{H}_{2} \mathrm{~S}$ at room temperature, and the lowest detection limits are as low as $20 \mathrm{ppb}$ and $50 \mathrm{ppb}$, respectively.

The sensing responses of metal oxide conductometric gas sensors can be explained by the processes of oxygen absorption and desorption onto the metal oxide surfaces [2]. Trapping of electrons at adsorbed molecules and band bending induced by these charged molecules are responsible for a change in the conductivity. When $\mathrm{O}_{2}$ mollecules are adsorbed onto the surface of metall oxides, they would extract electrons from the conduction band $E_{c}$ and trap the electrons at the surface in the form of ions which will lead to a bending of the band and an electron depleted region which can be described by

$\mathrm{O}_{2}+e \rightarrow n \mathrm{O}^{-}$

Reducing gases $\left(\mathrm{H}_{2} \mathrm{~S}, \mathrm{NH}_{3}, \mathrm{CO}\right.$, and $\left.\mathrm{H}_{2}\right)$ can react with these oxygen species, which will release the electrons back to the metal oxides and reverse the band bending. This results in an increased in conductivity. As described by

$$
\begin{aligned}
& \mathrm{H}_{2} \mathrm{~S}+3 \mathrm{O}^{-} \rightarrow \mathrm{H}_{2} \mathrm{O}+\mathrm{SO}_{2}+3 e \\
& 2 \mathrm{NH}_{3}+3 \mathrm{O}^{-} \rightarrow 3 \mathrm{H}_{2} \mathrm{O}+\mathrm{N}_{2}+3 e \\
& \mathrm{CO}+\mathrm{O}^{-} \rightarrow \mathrm{CO}_{2}+e \\
& \mathrm{H}_{2}+\mathrm{O}^{-} \rightarrow \mathrm{H}_{2} \mathrm{O}+e
\end{aligned}
$$

On the contrary, oxidizing gases like $\mathrm{NO}_{2}$ would decrease the device conductance. As described by this reaction:

$$
\mathrm{NO}_{2}+e \rightarrow \mathrm{NO}_{2}^{-}
$$


(a)
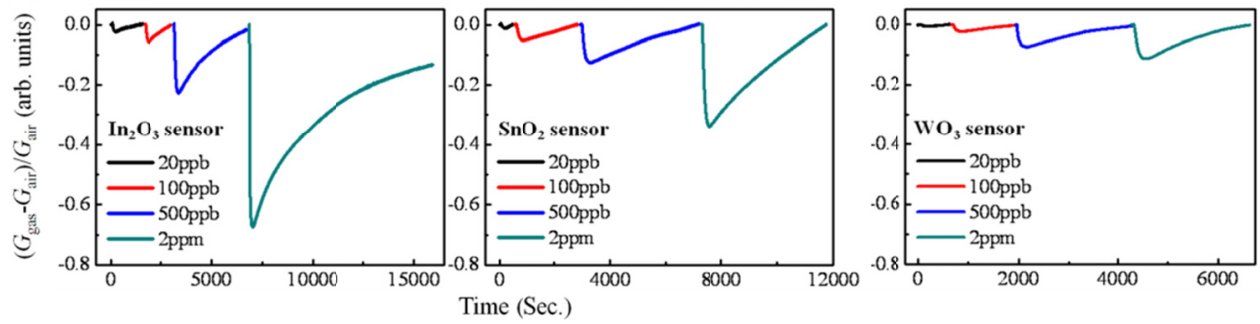

(b)
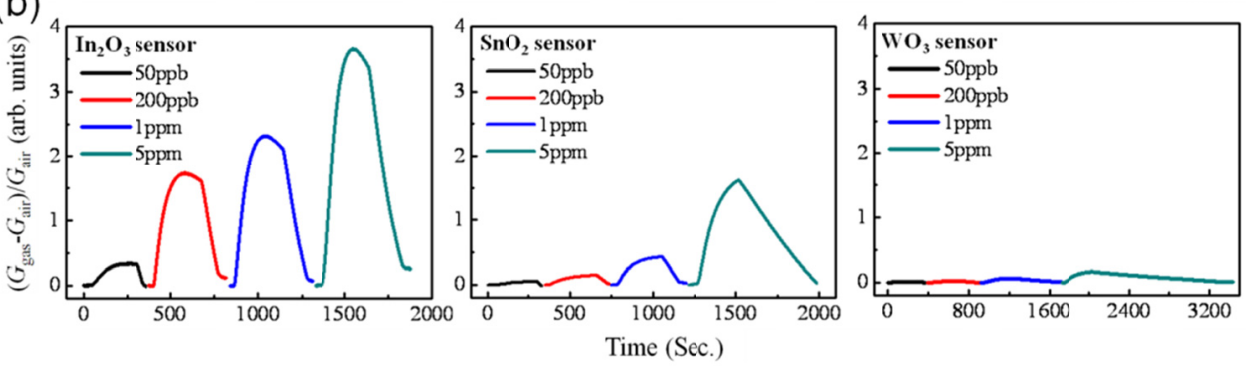

(c)
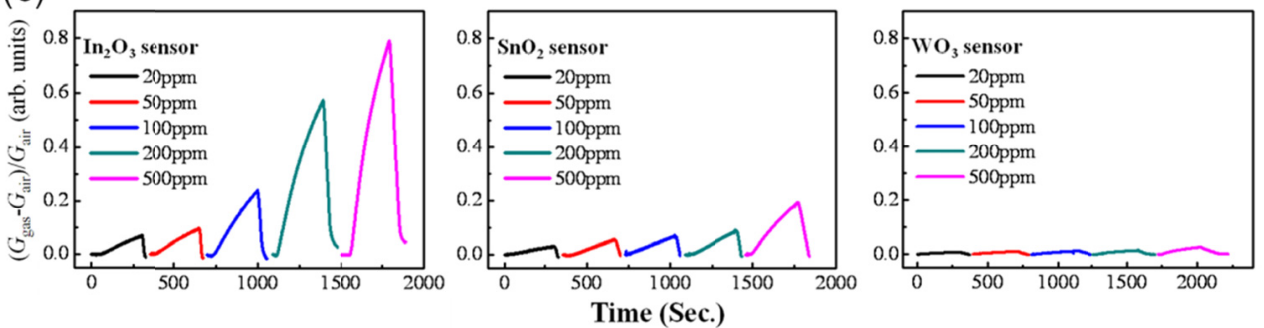

(d)
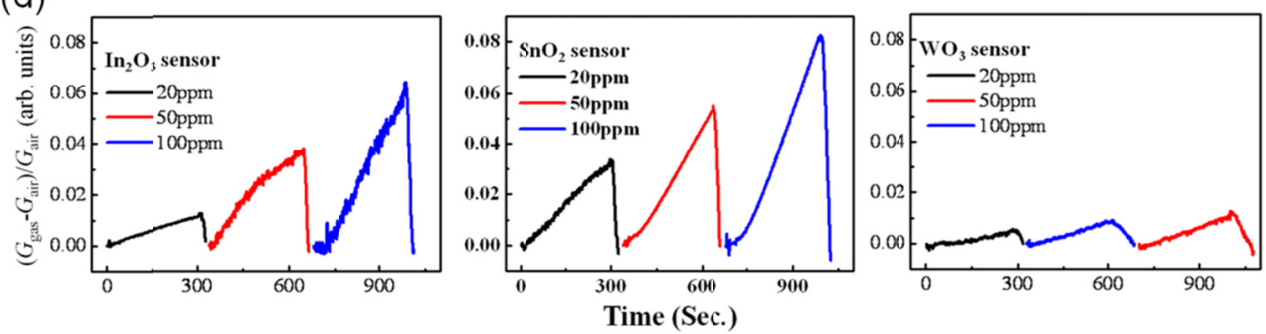

(e)
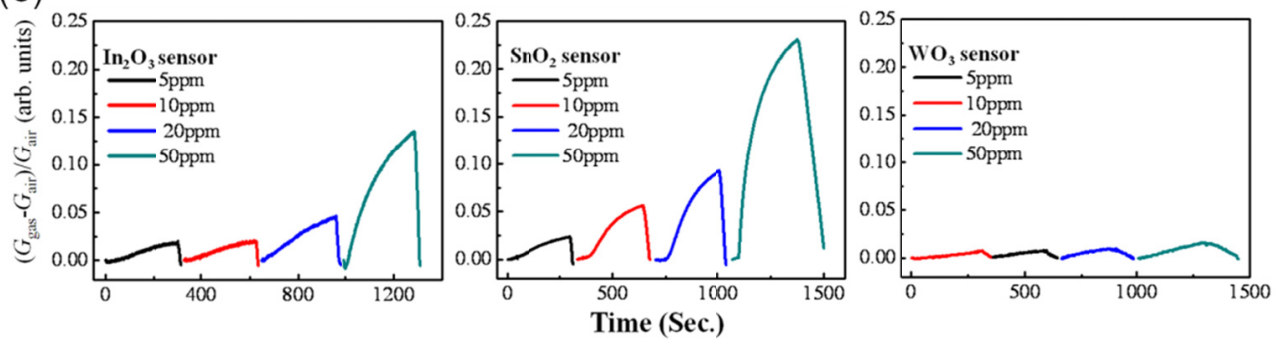

Fig.4. Sensing responses to different gases. (a) $\mathrm{NO}_{2}$; (b) $\mathrm{H}_{2} \mathrm{~S}$; (c) $\mathrm{H}_{2}$; (d) $\mathrm{CO}$; (e) $\mathrm{NH}_{3}$.

As one of the statistical methods, principal component analysis (PCA) is widely used to display the sensing responses of sensor arrays because it is a linear unsupervised method that requires little or no prior knowledge. Furthermore, in most cases only response variables (i.e. response strength, speed) are needed [11]. Fig. 4 is the PCA analysis of the sensing responses from the sensor arrays. Both $\mathrm{H}_{2} \mathrm{~S}$ and $\mathrm{H}_{2}$ are easily distinguished from the plot. Since $\mathrm{NO}_{2}$ is an oxidizing gas, it is readily picked up from the signal trend. So far, $\mathrm{NH}_{3}$ and 
$\mathrm{CO}$ are overlap at the low concentration region. However, by judging the response speed and sensitivity from Fig.4 (d) and (e), they can also be discriminated. We are currently exploring more metal oxide materials to discriminate them directly at low concentration.

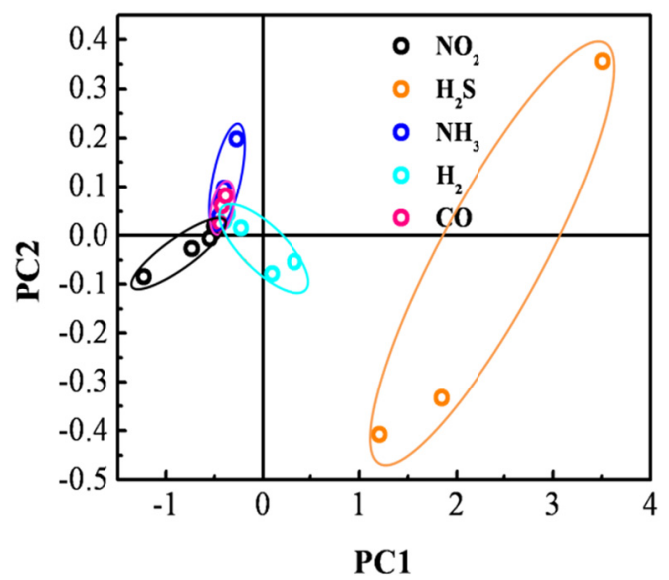

Fig. 4. PCA analysis of sensing responses from the sensor arrays.

\section{Conclusions}

An electronic nose system based on $\mathrm{ZnO}$ nanowire arrays with different gas sensing metal oxide coatings has been demonstrated for the gas discrimination of $\mathrm{NO}_{2}, \mathrm{H}_{2} \mathrm{~S}, \mathrm{H}_{2}, \mathrm{NH}_{3}$, and $\mathrm{CO}$ at room temperature assisted with principal components analysis (PCA). The $\mathrm{SnO}_{2}, \quad \mathrm{In}_{2} \mathrm{O}_{3}$, and $\mathrm{WO}_{3}$ coated $\mathrm{ZnO}$ sensors show responses to $\mathrm{NO}_{2}$ and $\mathrm{H}_{2} \mathrm{~S}$ with concentrations as low as $20 \mathrm{ppb}$ and $50 \mathrm{ppb}$, respectively. These results demonstrate a strong potential for highly sensitive sensor fabrication. Our method for engineering $\mathrm{ZnO} 3 \mathrm{D}$ nanowire arrays with different metal oxides provides a promising way to adapt more semiconductive materials on the novel route towards 3-D nanosensors for various gas detections.

\section{Acknowledgements}

This work was supported by DARPA grant No.HR0011-07-1-0032 and research grants from Louisiana Board of Regents Contract Nos. LEQSF(2007-12)-ENH-PKSFI-PRS-04, LEQSF (2008-11)-RD-B-10, and LEQSF(2011-13)-RDB-08.

\section{References}

[1] G. Shen , P. Chen , K, Ryu, C. Zhou, Devices and chemical sensing applications of metal oxide nanowires, J. Mater. Chem., 19, 828-839 (2009); doi: 10.1039/B816543B

[2] C. Wang, L. Yin, L. Zhang, D. Xiang, R. Gao, Metal oxide gas sensors: sensitivity and influencing factors, sensors, 10, 20882106(2010); doi:10.3390/s:100302088

[3] A. Kolmakov, M. Moskovits, Chemical sensing and catalysis by one-dimensional metal-oxide nanostructures, Annual Review of Materials Research 34, 151-180 (2004); doi:10.1146/annurev. matsci.34.040203.112141

[4] B. Cao, J.Chen, X. Tang, W. Zhou, Growth of monoclinic $\mathrm{WO}_{3}$ nanowire array for highly sensitive $\mathrm{NO}_{2}$ detection, J. Mater. Chem., 19, 2323-2327(2009), doi: 10.1039/B816646C

[5] D. Zhang, Z. Liu, C. Li, T. Tang, X. Liu, S. Han, B. Lei, C. Zhou, Detection of $\mathrm{NO}_{2}$ down to ppb levels using individual and multiple $\ln _{2} \mathrm{O}_{3}$ nanowire devices, Nano Letters 4, 1919-1924 (2004); doi: $10.1021 / \mathrm{nl} 0489283$

[6] K. Yao, D. Caruntu, Z. Zeng, J. Chen, C. O'Connor, W. Zhou, Partts per billion-level $\mathrm{H}_{2} \mathrm{~S}$ detection at room temperture based on selfassembled $\ln _{2} \mathrm{O}_{3}$ nanoparticles, J. Phys. Chem. C 113, 1481214817 (2009); doi: 10.1021/jp905189f

[7] K. Albert, N. Lewis, C. Sichauer, G. Sotzing, S. Stitzel, T. Vaid, D. Walt, Cross-reactive chemical sensor arrays, Chem. Rev. 100, 2595-2626 (2000); doi: 10.1021/cr980102w

[8] J. Chen, K. Wang, W. Zhou, Vertically aligned $\mathrm{ZnO}$ nanorod arrays coated with $\mathrm{SnO}_{2} /$ noble metal nanoparticles for highly sensitive and selective gas detection, IEEE Transactions on Nanotechnology 10, 968-974 (2011); doi: 10.1109/TNANO.2010.2091423

[9] L. Vayssieres, Growth of arrayed nanorods and nanowires of $\mathrm{ZnO}$ from aqueous solutions, $A d v$. Mater. 15, 464-466 (2003); doi: 10.1002/ adma. 200390108

[10] S. Xu, Y. Wei, Z. Wang, Patterned growth of vertically aligned $\mathrm{ZnO}$ nanowire arrays on inorganic substrates at low temperature without catalyst, J. Am. Chem. Soc., 130, 1495814959(2008), doi: 10.1021/ja806952j

[11] S. Jha, R. Yadava, Pattern recognition methods for SAW sensor array based electronic nose, 2011 IEEE, 1-4(2010), doi: 10.1109/ICCIC. 2010.5705774 